\title{
Guideline controversy
}

A re clinical practice guidelines unduly influenced by the financial associations and competing interests of the experts who write them? The lead editorial of our Nov. 22 issue was stimulated by a news release we had received from the Canadian Diabetes Association (CDA) that a criticized the Common Drug Review (CDR) for not approving for provincial formulary listing a new long-acting insulin that the CDA had recommended in its clinical-practice guidelines. According to the CDA, the CDR's judgment was uninformed because no clinical experts were involved. In reacting to this statement, we discovered that neither the CDA's nor the CDR's expert panels revealed or even dis- cussed potential financial conflicts of interest among the experts who were making the recommendations.

In this trio of articles, the CDA and CDR explain their guideline and recommendation processes and their rationales for not revealing authors' conflicts of interest. We also asked Dave Davis to comment more generally on the problem of producing unbiased clinical practice guidelines and solutions that are being implemented to improve guideline quality.

\section{John Hoey}

Editor, $C M A J$

\section{The Canadian Diabetes Association guidelines: putting the evidence first}

\author{
Michael C. Howlett, Donna Lillie
}

$\infty$

See related articles pages 335 and 337

I $\mathrm{n}$ a recent editorial ${ }^{1}$ that focused on the relationship between guideline authors and industry, $C M A J$ singled out the Canadian Diabetes Association (CDA) for considerable criticism. Some of the points raised are helpful in efforts of organizations such as ours to optimize guideline development processes. However, the editorial contained a number of important misstatements and errors. Physicians and others who rely on $C M A J$ to provide a thoughtful and balanced analysis of health care issues deserve an accurate and complete picture.

The CDA published clinical practice guidelines in 2003. ${ }^{2}$ The process for developing these guidelines, as was described in detail in the methods chapter (available: www.diabetes.ca - /cpg2003/downloads/methods.pdf), was rigorous and sys垈 tematic. In brief, the process involved exhaustive reviews of the literature, grading of the evidence and development of clinically directive evidence-based recommendations. In circumstances where no trial evidence had been published, consensus recommendations were required, demanding 100\% agreement among the members of the steering committee.
This work was undertaken by 62 nationally recognized experts who volunteered many thousands of hours to the project and received no financial compensation. To guard against all forms of bias, several measures were in place. Each volunteer completed a full duality-of-interest disclosure, which remains on file at the CDA. Clear policies on the disclosure of potential dualities and conflicts were explicit throughout the guideline process. Furthermore, during guideline discussions and deliberations, individuals were required to state any relevant potential dualities before presenting an opinion. After presentation at the annual professional conference of the CDA and before publication, the guidelines were reviewed by over roo external reviewers from Canada, the United States and Britain. As a final step, 3 methodologists reviewed each graded recommendation to ensure that the wording could be supported by the cited references and had been correctly graded. The CDA guidelines have been internationally acclaimed, ${ }^{3}$ and their process of explicitly stating the evidence for each recommendation is one of the most transparent in the world. 